\title{
Strongyloides stercoralis and Relatives: Recent Advances in General and Molecular Biology
}

\author{
James B. Lok
}

Published online: 25 September 2014

(C) Springer International Publishing AG 2014

\begin{abstract}
Human strongyloidiasis is a threat to global health, presenting significant challenges in diagnosis and clinical management. The imperative to incorporate strongyloidiasis more fully into control programs for soil-transmitted helminths is increasingly recognized. The unique life cycles of Strongyloides stercoralis and congeneric species contain both free-living and parasitic generations, and transcriptomic methods have recently identified genes of potential importance to parasitism in these worms. Proteomics recently revealed stage-specific secreted proteins that appear crucial to the host-parasite interaction. A comprehensive genome sequencing project for Strongyloides spp. is now nearing completion. Recent technical advances in transgenesis for $S$. stercoralis and $S$. ratti, including the first establishment of stable transgenic lines, promise to advance functional evaluations of genes expressed in conjunction with crucial life-cycle events. Studies employing these methods recently bolstered the hypothesis that $S$. stercoralis uses cellular signaling pathways homologous to three that regulate dauer larval development in Caenorhabditis elegans to regulate morphogenesis and development of its infective third-stage larva. The freeliving generation of Strongyloides makes classical genetics formally possible. Recent advances, such as a genetic map of $S$. ratti and a molecular genetic and karyotypic analysis of sex determination in $S$. papillosus, will greatly facilitate this approach. Advanced methods for study of chemosensation in C. elegans were recently applied to discover numerous hostattractant molecules that mediate host finding and contact by infective third-stage larvae of Strongyloides spp. Finally, nucleic acid-based diagnostic methods have recently come
\end{abstract}

\section{J. B. Lok $(\bowtie)$}

Department of Pathobiology, School of Veterinary Medicine,

University of Pennsylvania, 3800 Spruce Street, Philadelphia, PA 19104, USA

e-mail: jlok@vet.upenn.edu to the fore as alternatives to parasitological and immunodiagnostic techniques.

Keywords Strongyloides · Transcriptome · Genome · Proteome $\cdot$ Transgenesis $\cdot$ Genetics

\section{Introduction and Basic Biology}

Organismal Biology and Significance to Human Health

Strongyloides stercoralis is a significant human pathogen and also a parasite of dogs. Estimates of the human infection burden range from 30 to 100 million people, primarily in tropical and subtropical areas of sub-Saharan Africa, Southeast Asia, and South America [1]. It is likely that these are under-estimates of the true infection burden due to the poor sensitivity of diagnostic methods hinging upon on direct detection of larval S. stercoralis in patient stools. These will probably be revised upward as new molecular diagnostic methods for S. stercoralis infection (reviewed below) are incorporated into routine clinical practice $[2,3]$. Salient aspects of the S. stercoralis life cycle, such as the percutaneous route of infection by infective third-stage larvae (L3i) and their migration from the systemic circulation to alveoli of the lung and subsequently via the tracheae to the digestive tract, invite comparison to the life cycles of hookworms and, excluding the percutaneous route of infection, ascarid roundworms parasitizing humans and dogs [3]. Therefore, uncomplicated $S$. stercoralis infection predictably shares certain clinical manifestations with human and canine hookworm infection, including dermatitis at the point of L3i penetration, pulmonary signs such as dry cough and wheeze due to larval migration through the lung, and enteritis including epigastric pain, vomition, dysphagia and diarrhea [1]. However, the life history of S. stercoralis differs fundamentally from the life histories of other soil-transmitted nematodes in its ability to complete a full 
generation of free-living development outside the host and, with significant implications for pathogenesis, to undertake the process of autoinfection in which first-stage larvae elaborated by parasitic females develop precociously to autoinfective thirdstage larvae (L3a) in the intestines of the primary host (Fig. 1). These L3a then penetrate the gut of the primary host, migrate to the lungs via the systemic circulation and, via tracheal migration, establish a new generation of parasitic adults in the intestine. Sequential rounds of autoinfection may occur in an individual host. In immunocompetent hosts, autoinfective generations may occur at a low, well-regulated level, but in hosts immunocompromised by human T-cell lymphotropic virus 1 (HTLV-1) infection or by treatment with corticosteroids or other immunosuppressive drugs, sequential generations of autoinfection can result in geometric expansion of parasite numbers, invasion of multiple organ systems including lungs, liver and brain, and sepsis resulting from systemic release of enteric bacteria. Disseminated hyperinfections of this type may run a fatal course in 2-3 days [1, 3]. Low, well-regulated levels of autoinfection, allowing gradual replacement of senescent parasitic female worms, have been hypothesized as a mechanism

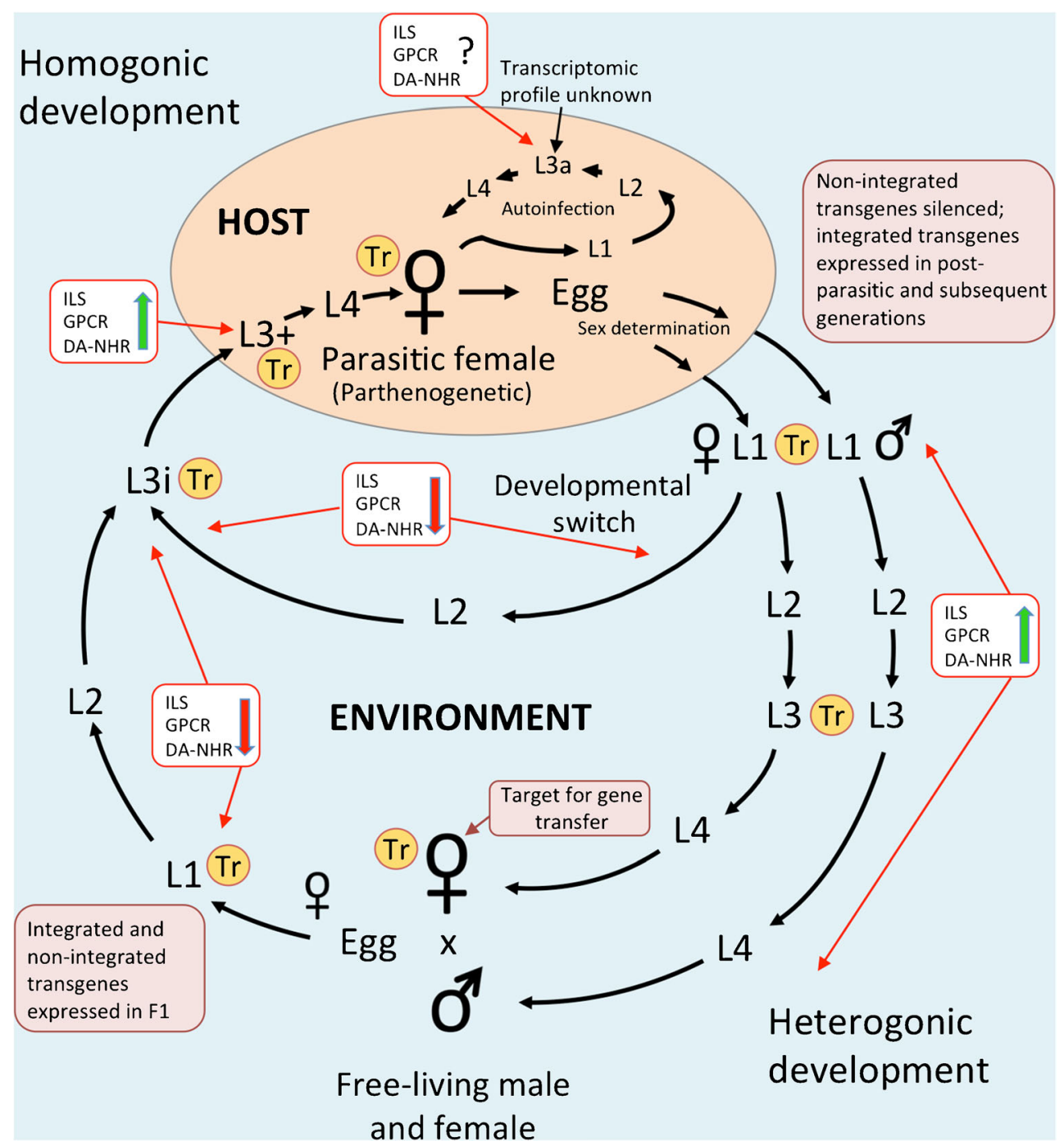

Fig. 1 Life cycle of Strongyloides stercoralis summarizing the current status of transgenesis, significant transcriptomic datasets, and hypothetical profiles of signaling through dauer-like regulatory pathways that are supported by current data. Yellow encircled $T r$ denotes a life stage for which transcriptomic datasets, stemming from RNA sequencing and/or microarray analysis, are available. Purple boxes indicate the free-living female as the effective target for delivery of transgene constructs by gonadal microinjection as well as the expression patterns of integrated and non-integrated transgenes before and after host passage. White boxes give the hypothesized status, based on the weight of current experimental and transcriptomic evidence, of three cellular signal transduction pathways with homologs that regulate dauer larval development in Caenorhabditis elegans: insulin-like signaling (ILS); G protein-coupled receptor (GPCR) signaling; dafachronic acid-nuclear hormone receptor (DAF-12) (DA-NHR) signaling. Green upward arrows indicate active signaling through the pathways; red downward arrows indicate downregulation of positive signaling or initiation of inhibitory signaling. F1 first filial generation, $L 1$ first-stage larvae, $L 2$ second-stage larvae, $L 3 a$ autoinfective third-stage larvae, $L 3 i$ infective third-stage larvae, $L 3+$ postinfective third-stage larvae, $L 4$ fourth-stage larvae 
by which immunocompetent hosts maintain exceedingly chronic, subclinical S. stercoralis infection, often for decades after the last exposure to L3i $[4,5]$. The literature is now replete with clinical reports of patients, frequently elderly migrants from endemic areas, in whom such exceedingly chronic subclinical infections progress to acute, sometimes fatal, disseminated hyperinfection as a result of treatment with corticosteroids or other immunosuppressive drugs [6].

Just as the host phase of the developmental cycle of $S$. stercoralis and its clinical manifestations invite comparison with infection by hookworms and other soil-transmitted nematodes, the environmental phase of this developmental cycle, which involves a single generation of free-living development with male and female adults (Fig. 1), invites comparison with both free-living nematodes and soil-transmitted parasitic species. It is therefore of significant interest from standpoints of comparative and evolutionary biology, which are reviewed in detail below. Importantly, crossing between adults of the free-living generation [7] affords S. stercoralis an opportunity for sexual recombination not present in the host phase in which only parthenogenetic females are present [8]. As is discussed below, the free-living generation of S. stercoralis and related species also allows both forward and reverse experimental genetics in this species at a level that is thus far impractical in other species of parasitic nematode $[9-12,13 \cdot 14 \cdot, 8,15,16]$.

\section{Evolutionary Biology: Strongyloides as a Model}

for the Evolution of Parasitism in Nematodes

The ability of S. stercoralis and congeneric parasites to undertake both obligately parasitic and free-living modes of life makes these worms apt subjects for studies of the molecular genetic requirements for vertebrate animal parasitism in nematodes and of the acquisition of these characteristics during evolution from free-living ancestors. It is worth noting that in their homogonic phases of development (Fig. 1), Strongyloides spp. are bona fide endoparasitic nematodes with life cycles that superficially resemble those of hookworms, with skin-penetrating L3i, post-parasitic larval stages that migrate somatically via pathways that include the lung, parasitic adults measuring some $2.0-2.8 \mathrm{~mm}$ in length, and, in the case of $S$. stercoralis, an adult lifespan of approximately 1 year in the host small intestine and progeny that are eliminated from the host in feces in the form of eggs or hatchling firststage larvae, which develop either directly or indirectly to L3i in fecally contaminated soil (Fig. 1). Strikingly, the same parasitic adult Strongyloides spp. can produce subsets of progeny that, upon elimination from the host, are fated to develop to a generation of males and females (Fig. 1) that have many of the basic biological attributes of obligately freeliving nematodes such as Caenorhabditis elegans. They live in the soil and feed on microbes that are abundant due to fecal contamination. They reproduce sexually and have a lifespan of 72-96 h. Therefore, the life cycle of $S$. stercoralis and of congeneric parasites contains two distinct adult morphs: one that is parasitic, long-lived, and parthenogenetic and another that is free-living, short-lived, and dioecious. These life cycles also contain two distinct patterns of larval development in the extrinsic environment. One of these, exhibited by larval progeny of parasitic females, involves sex determination (Fig. 1) and switching between homogonic (directly to L3i and then to parasitic females) and heterogonic (to L3i via a free-living generation of males and females) developmental fates by genetically female larvae (Fig. 1). It is noteworthy that genetically male larvae in this post-parasitic phase of the life cycle all develop to free-living adults (Fig. 1). The other program of larval development in the extrinsic environment occurs in the progeny of free-living males and females. In S. stercoralis, all of these progeny are genetically female, and all of them develop to L3i, which persist in the soil and continue their development only when they infect a susceptible mammalian host (Fig. 1). Therefore, the life cycle of S. stercoralis, and the life cycles of Strongyloides spp. generally, offer the opportunity to compare parasitic and non-parasitic morphs of selected life stages, such as parasitic and free-living females, and homogonically and heterogonically developing first- and third-stage larvae that arise from the same genome. The remarkable differences in body plan, ecological niche, and developmental fate that such comparisons reveal at the organismal level invite comparisons at the molecular level aimed ideally at determining patterns of gene expression that are unique to and possibly essential for establishing and maintaining the parasitic state. These are discussed further in the sections on developmental biology and comparative transcriptomics below.

Developmental Biology: Cellular Signaling in the Infective Process

Recent studies of developmental regulation during the infective process in $S$. stercoralis have been driven by the longstanding 'dauer hypothesis', which posits that cellular signaling pathways homologous to those regulating dauer larval development in C. elegans regulate the formation and further development of L3i in parasitic nematodes [17, 18]. This developmental progression in parasitic nematodes such as Strongyloides, filariae, and hookworms includes the crucial infective process in which environmental L3i resume their development upon invasion of a permissive mammalian host. Under the dauer hypothesis, these regulatory pathways would include a G protein-coupled receptor (GPCR) signaling pathway in amphidial neurons that transduces physicochemical cues of host approach and contact to regulate the synthesis of insulin-like peptides (ILPs) and transforming growth factor- $\beta$ (TGF $\beta$ )-like peptides, which regulate their respective signaling pathways in numerous tissues in the worm that are 
remodeled or function in morphogenesis and development of L3i. Parallel insulin- and TGF $\beta$-like signals would act in concert on a downstream dafachronic acid-nuclear hormone receptor signaling pathway to ultimately regulate development by L3i during the infective process. Recent findings have demonstrated that GPCR signaling is likely involved in developmental activation in L3i following host invasion as reflected by resumption of feeding by these larvae [19•] (Fig. 1). 8-bromo-guanosine 3',5'-cyclic monophosphate (8br-cGMP) stimulated feeding in a dose-dependent fashion by L3i cultured in the absence of host-like physicochemical cues such as elevated temperature $\left(37^{\circ} \mathrm{C}\right)$ and the growthpromoting components of Dulbecco's Modified Eagles Medium (DMEM). Notably, RNA sequencing (RNAseq) indicated that, concomitant with it's promotion of feeding, 8-brcGMP also stimulates patterns of abundance of ILP encoding transcripts $S s-i l p-5$ and $S s-i l p-6$ in L3i that are highly similar to those seen in L3i following $48 \mathrm{~h}$ of development in susceptible gerbils [20 ${ }^{\bullet}$. These data are consistent with genetic evidence from C. elegans [21] that GPCR signaling acts upstream of insulin-like signaling (ILS) to regulate the development of dauer third-stage larvae.

Building upon earlier findings on the insulin-regulated transcription factor, Ss-DAF-16 (originally designated FKTF-1) [22], further evidence that ILS regulates L3i development (Fig. 1) appeared recently in a study confirming that $S$. stercoralis has conserved the insulin-regulated phosphoinositol-3-kinase (PI3K) encoded in C. elegans age1 and that the PI3K inhibitor LY294002 can block resumption of feeding by L3i grown under host-like culture conditions [23]. A full molecular characterization of the insulin receptor kinase encoding gene $S s-d a f-2$ was recently completed [24] and included abundance profiles of its two encoded isoforms in seven life stages of $S$. stercoralis. This study employed transgenesis to identify the intestine as the primary site of $S s$ daf-2 expression.

Recent studies [19•] also tested the hypothesis that ILS acts upstream to promote dafachronic acid Ss-DAF-12 signaling in S. stercoralis. Dafachronic acid-DAF-12 signaling promotes continuous reproductive development and, presumably, dauer recovery in C. elegans [25]. Prior studies had demonstrated that the DAF-12 nuclear hormone receptor is conserved in S. stercoralis and in Ancylostoma caninum, and that exogenously applied $\Delta 7$-dafachronic acid ( $\Delta 7$-DA) stimulates post-infective development by L3i of both parasites at midnanomolar concentrations, similar to its activity range in C. elegans [26]. Data from studies of genetic epistasis [27] and experimental data showing that exogenous $\Delta 7$-DA suppresses a dauer constitutive loss-of-function mutation in the insulin receptor kinase DAF-2 [25] all support the hypothesis that in C. elegans dafachronic acid-DAF-12 signaling regulates dauer larval development downstream of ILS. Surprisingly, however, a kinetic comparison of developmental activation by $S$. stercoralis L3i in response to $200 \mu \mathrm{M}$ 8-brcGMP and $400 \mathrm{nM} \Delta 7$-DA showed that $\Delta 7$-DA induces the response in cultured L3i more rapidly than 8-br-cGMP. Also contrary to expectations based on the C. elegans paradigm, RNAseq conducted on populations of $S$. stercoralis $\mathrm{L} 3 \mathrm{i}$ that had been treated with $400 \mathrm{nM} \Delta 7$-DA revealed patterns of $S s$ ilp-5 and Ss-ilp-6 transcript abundance that were virtually identical to those induced in S. stercoralis L3i by treatment with $200 \mu \mathrm{M}$ 8-br-cGMP and by developmental activation in a susceptible mammalian host. Results of a third experiment were also contrary to expectations under the $C$. elegans paradigm in showing that treatment with $400 \mathrm{nM} \Delta 7$-DA fails to override tenfold reduction in the feeding response imposed on cultured S. stercoralis L3i by the PI3k inhibitor LY294002 [19•]. Overall, recent findings further support the conclusion that GPCR signaling, ILS, and dafachronic acid Ss-DAF-12 signaling all regulate developmental activation of $S$. stercoralis L3i during the infective process (Fig. 1). Coupled with findings on developmental trends in abundance of transcripts encoding TGF $\beta$-like ligands in S. stercoralis, they also support the recent assessment by Crook [17] that the weight of current evidence supports the 'Dauer Hypothesis' as it pertains to GPCR, insulin-like, and dafachronic acid-DAF12 signaling in parasitic nematodes, but not as it pertains to TGF $\beta$ signaling, which appears to act in parasitic nematodes in direct opposition to what would be predicted under this hypothesis, prompting the alternate interpretation that TGF $\beta$ like ligands, which in most cases are strongly upregulated in L3i, may serve an immunomodulatory function protecting these invasive larvae as they begin their interactions with the host following infection.

Assembly of the vulva in young parasitic females is crucial to oviposition and therefore to propagation of Strongyloides spp. in the environment. A recent histochemical investigation of vulvar assembly in S. venezuelensis [28] underscored a central role for the cytoskeleton, and most specifically filamentous actin in this process.

\section{Developmental Biology: Sex Determination}

As recently reviewed [12], parasitic female Strongyloides spp. reproduce by mitotic parthogenesis, making individuals in the post-parasitic generation genetically identical to their mothers. Strikingly, there is sex determination in this generation such that a proportion of progeny becomes genetically male and develops to free-living adult males, and a proportion remains genetically female and capable of switching between development directly to L3i, which subsequently invade the host, or to free-living females (Fig. 1). Recent findings on the effect of environmental temperature on the infectivity of S. venezuelensis larvae in mice [29] probably reflects the practical consequences of skewing this switching event towards direct development to the L3i by elevated temperature. 
Karyotypes of $S$. ratti and S. stercoralis consist of two pairs of autosomes and a pair of sex (X) chromosomes in females and a single $\mathrm{X}$ chromosome in males, all chromosomes being of medium length, reflecting an XX/XO sex determination. By contrast, karyotypes of $S$. papillosus have been alternatively characterized as consisting either of a pair of long (L) chromosomes and a pair of medium length (M) chromosomes or of a single $\mathrm{L}$ chromosome, three $\mathrm{M}$ chromosomes, and a single short (S) chromosome. Nemetschke et al. [12] have recently used molecular genetic techniques to confirm previous speculation that the regions homologous to the $\mathrm{X}$ chromosomes of $S$. ratti account for the elongation of $\mathrm{L}$ chromosomes of S. papillosus and that conflicting descriptions of the karyotype of $S$. papillosus reflect observations before and after a chromatin diminution event giving rise to a male karyotype of 1L3M1S and a female karyotype of 2L2M. Characterization of the eliminated chromosomal region in $S$. papillosus revealed that it contains many genes [12], in contrast to the chromatin diminution observed in Ascaris spp. where eliminated chromosomal regions contain a majority of non-coding DNA. Most significantly, Nemetschke et al. [12] observed that remains of the $\mathrm{L}$ chromosome undergoing diminution are excluded from mature sperm in $S$. papillosus, thus functionally reconstituting the $\mathrm{XX} / \mathrm{XO}$ sex determination system seen in the related species $S$. ratti and $S$. stercoralis. Further commentary on this unique sex determination system in S. papillosus and the accompanying general analysis of sex determination in Strongyloides spp. was published contemporaneously [30].

\section{Host-Parasite 'Communication'}

Since its L3i infect the host percutaneously, S. stercoralis and its congeners have evolved a set of behaviors that increase the probability of host contact as well as sensitivity to a multiplicity of attractive olfactory cues emanating from their respective mammalian hosts. Previously, the only such chemoattractant to have been characterized at the molecular level was urocanic acid, a common component of human and other mammalian skin [31]. More recently, Castelletto et al. [32] have undertaken an extensive comparison of phylogenetically diverse parasitic nematodes, including entomoparasitic species as well as mammalian parasites in which L3i infect the host by active skin penetration (S. stercoralis, S. ratti, and Nippostrongylus braziliensis) or by passive ingestion (Haemonchus contortus). Overall, host preference appears to be a stronger determinant of specific olfactory sensitivities than phylogeny among the parasitic nematodes studied. For example, L3i of two distantly related rodent parasites, $S$. ratti and $N$. braziliensis, responded to subsets of olfactory cues that were more similar to each other than to the subsets attracting L3i of S. stercoralis. In general, L3i of $S$. stercoralis were characterized as fastmigrating 'cruisers', which increase the probability of host contact by continuous spontaneous movement across horizontal substrates. By contrast, L3i of the passively ingested $H$. contortus were characterized as 'ambushers', which exhibit little unstimulated migration but rather ascend blades of grass where they are more liable to be ingested by their grazing ruminant hosts. L3i of $S$. stercoralis were attracted to a wide range of humanassociated odorants including several of the most active ones, such as 3-heptanol, 1-nonanol and both 2- and 3methyl-1-butanol, that are also strong chemoattractants for host-seeking mosquitoes. That these cues act as volatile odorants as opposed to aqueous gustatory stimuli was underscored by the fact that the L3i responded to them when they were spotted on the inner side of the assay plate lid as well as when they were applied to the agar layer upon which the worms migrated.

In addition to the direct chemical communication between host and parasite that mediates host finding and contact by L3i, there is substantial evidence (reviewed in Streit [33]) that parasitic females of $S$. ratti, S. ransomi are induced to produce greater proportions of male progeny and a population of female progeny with an increased tendency towards heterogonic (free-living) development in response to a mounting protective immune response by the host. It is striking, therefore, that microarray analyses reveal only modest $(<3$-fold) differences in transcript abundance between parasitic female $S$. ratti recovered at 5 and 9 days of infection from naïve rats and from rats that had been immunized by drug-abbreviated infection [34]. Prominent among the few transcripts upregulated in the presence of the host immune response were those encoding homologs of UNC-87 and UNC-54 in C. elegans, both proteins related to muscle function. These findings are certainly consistent with the repair of muscle in the parasitic female damaged by the immune response, as discussed by the authors, but their relation to altered sex determination and developmental fate among the progeny remains obscure. The increased sensitivity and resolution enabled by RNAseq, relative to microarray analysis, may help to clarify the molecular basis for this profound influence of host immunity on subsequent developmental fate in the progeny of surviving parasitic females.

\section{Studies at the Genomic, Transcriptomic, and Proteomic Scales}

Genome Sequences

At the time of writing, draft genome sequences for $S$. stercoralis, the congeners $S$. ratti, S. papillosus and $S$. venezuelensis, and the related outgroup Parastrongyloides trichosuri are currently under annotation by collaborators at the Wellcome Trust Sanger Center (Hinxton, Cambridge, 
UK). Current versions of these draft genome sequences are available to the public via file transfer at ftp://ftp.sanger.ac.uk/ pub/project/pathogens/HGI/.

The genome of $S$. stercoralis comprises 43 megabases (mb) and 13,114 genes. In general the $S$. stercoralis genome has significantly fewer and smaller introns than $C$. elegans and parasitic nematodes representing Clades I, III, and V [35]. For example, the $S$. stercoralis genome contains 21,058 introns $(9.4 \%$ of the total genomic sequence) with a median length of 52 bp. By contrast, $H$. contortus, in Clade V along with $C$. elegans, has 171,763 introns (27.7\% of the genome) with a median length of $168 \mathrm{bp}$. Comparable data for Ascaris suum in Clade III are 100,624 introns comprising $37.7 \%$ of the genome with a median length of $690 \mathrm{bp}$. These findings suggest a rapid loss of introns during the evolution of parasitic nematodes such as $S$. stercoralis in Clade IV.

\section{The Transcriptome}

High-capacity or 'next-generation' sequencing technology has enabled the recent creation of some robust transcriptomic databases for $S$. stercoralis. One of these, reported by Stoltzfus et al. [20•], contains sequences of polyadenylated RNA from seven life stages of the parasite selected for their key roles in the infective process, in the maintenance of parasite populations in the host and external environment, and in the property unique to Strongyloides and related Clade IV parasites of developmental programming for either free-living or parasitic fates. These stages are actively reproducing free-living females (FL Females), post-free living firststage larvae (PFL L1), L3i, post-infective third-stage larvae $(\mathrm{L} 3+)$ recovered $48 \mathrm{~h}$ after inoculation into susceptible gerbils, gravid parasitic females (P Females), predominantly (95\%) heterogonically developing post-parasitic first-stage larvae (PP L1), and post-parasitic larvae, approximately third-stage, developing heterogonically to free-living adults and predominantly female (PP L3) (Fig. 1). High-quality sequences were derived by Illumina HiSeq technology from $100 \mathrm{bp}$ paired end reads from polyadenylated RNA libraries prepared in biological triplicate from each stage. This resulted in a total of 2.36 billion reads, of which $74 \%$ aligned to the $41 \mathrm{mb}$ of $S$. stercoralis genomic contigs available at the time of the study.

Another recent transcriptomic study of $S$. stercoralis L3i that employed next-generation sequencing techniques [36] emphasized prediction of proteins involved directly in the host parasite interaction as opposed to developmental regulatory molecules intrinsic to the parasite. Of the more than 11,000 proteins predicted by transcripts sequenced in this study, most were novel and were predicted to include an abundance of enzymes such as kinases and proteases and more than 1,200 excretory secretory proteins. Some of the proteins predicted in this study were potential drug candidates.
Next-generation sequencing has also been employed to create transcriptomic resources for other species of Strongyloides, such as $S$. venezuelensis [37, 38], which is used widely as a model for immunological studies. Altogether these studies encompassed four developmental stages: eggs/first-stage larvae (L1), environmental L3i, L3i derived from the host lung, and parasitic females [37, 38].

Valuable insights into the transcriptomic changes undergone by $S$. stercoralis at crucial life cycle transitions have also been gained recently through microarray analyses. Recent microarray-based studies of S. ratti, and earlier studies cited therein, stress comparisons of parasitic and non-parasitic morphs of the parasite [39] and also of parasites living in the environments of immune and naive hosts [34]. Ramanathan et al. [40] amassed microarray data from post-parasitic L1 and L3i of $S$. stercoralis that reveal patterns of differential gene expression associated with the acquisition of host infectivity during that transition. These, along with the biological conclusions of the other transcriptomic studies cited above, are discussed in the following section.

\section{Comparative Transcriptomics}

The overall rubric of discovering 'genes for parasitism' has driven a series of recent transcriptomic studies of developmental stages in Strongyloides spp., initially using profiles of expressed sequence tag (EST) abundance and protein expression $[41 \cdot, 42,43]$ and, more recently, microarray technology [40] and next-generation sequencing [36, 37, 34, 20•]. Studies focusing explicitly on detecting genes essential for parasitism in Strongyloides have taken the approach of identifying ESTs from $S$. ratti that do not have identifiable homologs in C. elegans and that are unique to or significantly upregulated in parasitic females relative to free-living females. This unbiased approach identified a panel of seven ESTs unique to or upregulated in parasitic females $[42,43]$. Subsequently, these results were confirmed by quantitative reverse transcriptase polymerase chain reaction (qRT-PCR) analysis for five of the ESTs, the genes characterized by bioinformatic analyses and the expression dynamics of their encoded mRNAs, and the encoded proteins were described for three candidates [41•]. Of the three parasitism-associated genes undergoing complete analysis in this study, two, SR00007 and SR00449, encode homologs of chondroitin proteoglycan 3 (CPG-3) in A. suum (Table 1). The protein encoded in one of these, SR00449, is expressed in parasitic females of $S$. ratti and is secreted into the worm's surroundings in the host. Transcripts encoding the other CPG-3 homolog, SR00007, are also present in parasitic female $S$. ratti, or in the eggs they carry in utero, but these transcripts are not expressed until the post-parasitic L1 hatch. A similar pattern of maternal or in utero transcription followed by translation in newly hatched post-parasitic L1 occurs in a third parasitism-specific gene in S. ratti, SR00984. This gene 
Table 1 Summary of genes, gene products, and functional pathways ascribed biological significance or potential as drug or vaccine targets based on transcriptomic, proteomic, or experimental findings highlighted in this review

\begin{tabular}{|c|c|c|c|c|c|}
\hline Species & Stage & Gene/gene product/pathway & Type of investigation & Putative function/application & Reference \\
\hline \multirow[t]{6}{*}{$\begin{array}{l}\text { Strongyloides } \\
\quad \text { ratti }\end{array}$} & \multirow[t]{2}{*}{ L3i } & $\begin{array}{l}\text { Astacin metalloprotease } \\
\text { L3 Nie antigen }\end{array}$ & \multirow[t]{4}{*}{ Proteomic } & $\begin{array}{l}\text { Host tissue invasion } \\
\text { Immunogenic; vespid wasp } \\
\text { venom family }\end{array}$ & \multirow[t]{3}{*}{$50 \bullet$} \\
\hline & & Fatty acid retinol binding protein & & Scavenging host lipids & \\
\hline & \multirow[t]{4}{*}{ P Female } & Prolyl oligopeptidase & & Motility/survival & \\
\hline & & $\begin{array}{l}\text { Sra-HSP-17.1 } \\
\text { Sra-HSP-17.2 }\end{array}$ & & $\begin{array}{l}\text { Binds host epithelium; elicits } \\
\text { IL-10 production } \\
\text { Elicits IL-10 production }\end{array}$ & {$[51]$} \\
\hline & & $\begin{array}{l}\text { SR00007 chondroitin proteoglycan } \\
\text { SR00449 chondroitin proteoglycan }\end{array}$ & \multirow[t]{2}{*}{ Transcriptomic/EST } & $\begin{array}{l}\text { Immunogen; function unknown } \\
\text { Secreted immunogen; function } \\
\text { unknown }\end{array}$ & \multirow[t]{2}{*}[41\cdot]{} \\
\hline & & SR00984 small HSP & & Immunogen; function unknown & \\
\hline \multirow[t]{3}{*}{ S. stercoralis } & \multirow[t]{3}{*}{ L3i } & $\begin{array}{l}\text { Ss-age-1 (ILS) } \\
\text { GPCR signaling } \\
\text { Dafachronic acid-Ss-DAF-12 signaling }\end{array}$ & Experimental & $\begin{array}{l}\text { All promote developmental activation } \\
\text { of cultured L3i in the absence of } \\
\text { host-like stimuli }\end{array}$ & {$[19 \bullet]$} \\
\hline & & $\begin{array}{l}\text { HSP-90 } \\
\text { Cytochrome oxidase ucr } 2.1 \\
\text { Sperm containing glycoprotein domain }\end{array}$ & \multirow[t]{2}{*}{ Transcriptomic/microarray } & $\begin{array}{l}\text { Potential chemotherapeutic target } \\
\text { Potential vaccine targets }\end{array}$ & \multirow[t]{2}{*}[40]{} \\
\hline & & Fatty acid retinol binding protein-1 & & Scavenging host lipids & \\
\hline \multirow[t]{2}{*}{ S. venezuelensis } & P Female & Ferrochetalase & \multirow[t]{2}{*}{ Transcriptomic/RNAseq } & Iron porphyrin catabolism & {$[37][38][49]$} \\
\hline & LL3 & Multiple transcripts & & Secretory glycan biosynthesis & {$[37][38]$} \\
\hline S. papillosus & L3i & $\begin{array}{l}\text { Spa-asp-2 aspartic protease } \\
\text { Spa-lys lysozyme }\end{array}$ & Experimental & $\begin{array}{l}\text { Both upregulated at skin } \\
\text { penetration }\end{array}$ & {$[45]$} \\
\hline
\end{tabular}

$E S T$ expressed sequence tag, GPCR G protein-coupled receptor, $H S P$ heat shock protein, $I L-10$ interleukin-10, $I L S$ insulin-like signaling, $L 3 i$ infective third-stage larva, $L L 3$ post-infective third-stage larvae collected from lungs of infected mice $72-75 \mathrm{~h}$ after inoculation of L $3 \mathrm{i}, P$ Female parasitic female, RNAseq RNA sequencing

has significant homology to those encoding small heat shock proteins (HSPs) in Trichinella pseudospiralis and in C. elegans (Table 1). All three of the parasitism-associated gene products that were characterized by Spinner et al. [41•] are recognized by antibodies in the sera collected from rats in the early phase of $S$. ratti infection, prompting the question of whether they may induce a protective response in this host, which effectively suppresses egg output by $S$. ratti within the first 25 days of infection [44]. Although preliminary data suggest that immunization with one or more of these parasitism-associated proteins may decrease the reproductive output of $S$. ratti, further experimentation is required to conclude this $[41 \bullet]$. Nevertheless, this approach demonstrates the power of unbiased transcriptomic screening to identify parasitism-associated gene products for follow-up evaluation as targets for intervention with novel drugs or vaccines. Microarray analysis of transcriptomic differences between post-parasitic L1 and L3i of S. stercoralis [40] revealed upregulation of transcriptional 'machinery' in the L1, as would be expected in these rapidly differentiating larvae. Among the transcripts increasing in abundance in the L3i were those encoding the potential chemotherapeutic targets HSP-90 and cytochrome oxidase ucr 2.1, an ortholog of the fatty acid retinol binding protein that formed the basis of an effective vaccine against $A$. ceylanicum and the sperm-containing glycoprotein domain that constitutes the target of a vaccine against Cooperia punctata, an important trichostrongyle parasite of ruminant livestock. These findings provide a sound rationale for exploring these chemotherapeutic and vaccine strategies in human strongyloidiasis.

Adopting a more hypothesis-driven approach, Biewener et al. [45] used targeted qRT-PCR to demonstrate that in S. papillosus, transcripts encoding an aspartic protease, SpaASP-2 are upregulated in L3i during the process of skin penetration, and those encoding the lysozyme Spa-LYS are downregulated at this crucial point in the infective process. The hypothesis that these transcripts would be regulated at the point of percutaneous migration was based on findings that one isoform of a homologous aspartic protease is preferentially expressed in parasitic females of $S$. ratti [46] and that similar lysozymes are among the excretory-secretory products of the hookworm A. caninum [47].

While the transcriptomes resulting from the RNAseq study by Stoltzfus et al. [20 ${ }^{\bullet}$ represent a wealth of information on developmentally regulated genes affecting all aspects of the biology of $S$. stercoralis, the study itself emphasized 
abundance profiles of transcripts homologous to genes in four cellular signaling pathways that regulate dauer larval development in C. elegans, this being a hypothetical framework for regulation of L3i development in parasitic nematodes [18]. Some findings, such as the upregulation of specific GPCR signaling elements in the L3i were expected given the necessity of this larval stage detecting chemical and thermal cues from the host. Also expected was the presence of ILPs that are coordinately regulated during development of L3i of S. stercoralis. A surprising finding in this aspect of the study was the marked reduction in the number of transcripts encoding different ILPs, from 40 in C. elegans to only seven in S. stercoralis. Four of the seven S. stercoralis ILPs may be categorized by whether their transcript abundance rises or falls during post-infective developmental activation of L3i in the host, patterns that would be consistent with agonists or antagonists, respectively, of Ss-DAF-2, the ortholog of the dauer regulatory insulin-like receptor kinase in C. elegans [48]. Findings from the RNAseq study by Stoltzfus et al. [20 $]$ confirmed previous observations that transcripts encoding homologs of the TGF $\beta$-like ligand DAF-7, which suppresses dauer formation in $C$. elegans, are actually most abundant in $\mathrm{L} 3 \mathrm{i}$, a pattern that is inconsistent with conservation of C. elegans-like DAF-7 function in Strongyloides. Finally, transcripts encoding $S$. stercoralis homologs of enzymes catalyzing biosynthesis of dauer-regulatory steroids, dafachronic acids, in C. elegans [25] were not coordinately regulated during L3i development.

Salient among the transcriptomes of four key life stages of S. venezuelensis $[37,38]$ are transcripts encoding an expanded repertoire of astacin-like zinc metalloproteases, and a ferrochelatase unique to animal parasitic nematodes, which may catalyze the reverse of the ultimate reaction in heme biosynthesis to scavenge iron from host porphyrins [49]. Also discovered in S. venezuelensis was a cluster of transcripts, upregulated in lung-derived L3i, that encode enzymes catalyzing biosynthesis of secreted glycans that are likely to be important in interactions with the intestinal epithelium of the host [37, 38].

\section{The Proteome}

At the time of writing, systematic proteomic studies of $S$. stercoralis have not been undertaken. By contrast, the proteome of $S$. ratti, particularly the secreted proteome or " secretome', is under active investigation [50•, 51], with particular emphasis on excretory/secretory proteins from the parasite that could modulate immune or other defensive mechanisms in the host intestinal mucosa. The secretome of $S$. ratti comprises 586 proteins, of which 196 are secreted exclusively by L3i [50॰]. Prominent in the secretome of L3i are an astacin metalloprotease, likely used to penetrate the host skin, the L3 Nie antigen, and a fatty acid retinoid binding protein (Table 1).
Among the 79 proteins secreted exclusively by parasitic females are a prolyl oligopeptidase, some small HSPs, and a secreted acidic protein. Free-living stages of $S$. ratti uniquely secrete 35 proteins, including a lysozyme, a carbohydratehydrolyzing enzyme, and a saponin-like protein. Most significantly, Soblik et al. [50•] showed that small-molecule inhibitors of the secreted prolyl oligopeptidase suppressed motility of cultured parasitic female $S$. ratti in a dose-dependent fashion, within the low micromolar range, providing evidence that this protein is essential for survival of these stages. Two homologs of C. elegans HSP-17, Sra-HSP-17.1 and SraHSP-17.2, which are secreted by parasitic female $S$. ratti, are immunogenic in infected rats, and Sra-HSP-17.1 binds to cultured rat epithelial cells, suggesting a role for this protein in the host parasite interaction. Host monocytes exposed to either Sra-HSP produced interleukin-10 but not tumor necrosis factor- $\alpha$, suggesting a role for these proteins in directing the host immune response (Table 1). In general, these recent studies $\left[50^{\bullet}, 51\right]$ underscore the power of the proteomic approach in identifying potential targets for immunological or chemotherapeutic intervention. Transcriptomic data arising from next-generation sequencing provides a wealth of data that could be used to predict the secretomes of Strongyloides spp. A semi-automated computational approach to this process has been developed [52] and verified in S. ratti by comparing predictions derived in silico to known profiles of excretory-secretory proteins in this parasite.

\section{Linkage Mapping}

Classical genetic methods, which depend to a large degree on the ability to establish experimental crosses between individuals differing in a trait of interest, have not been used to any significant degree in animal parasitic nematodes because adult males and females of most species are, in practical terms, inaccessible due to their obligate endoparasitic association with the host. One of the great advantages of Strongyloides spp. as an experimental model for parasitic nematodes is that all species in the genus are capable of developing through at least one generation of free-living male and female worms. Genetic exchange between these males and females on mating has been confirmed for $S$. ratti [7] and likely pertains throughout the genus. This makes Strongyloides spp. the only group of animal parasitic nematodes in which genetic crosses are practical, and therefore the only likely subjects for classical genetic study among this important group of pathogens. One prerequisite for locating and ultimately identifying genetic loci encoding traits of interest through crossing is a genetic linkage map. Nemetschke et al. [13•] recently generated such a genetic map for $S$. ratti by crossing two isofemale lines and backcrossing the progeny to create an F2 in which genetic linkage of 74 molecular markers was analyzed. This analysis generated a map predicting three linkage groups, consistent 
with chromosome number in $S$. ratti, and having an average density of one marker per $1.27 \mathrm{cM}$. The map also correctly predicts the $\mathrm{XX} / \mathrm{XO}$ pattern of sex determination in free-living $S$. ratti adults [12]. This map will greatly facilitate classical, and ultimately molecular genetic, analysis of crucial traits such as anthelmintic resistance in $S$. ratti, and, by proxy, in other animal parasitic nematodes. The power of such a genetic map as a tool for analyzing genetic cross-data and defining the molecular basis of an anthelmintic (oxamniquine) resistance trait through classical genetics was recently demonstrated in the parasitic trematode Schistosoma mansoni [53].

\section{Functional Genomic Methods}

The same limitations in accessibility to reproductive adult animal parasitic nematodes that hamper classical genetic study have also limited the application of modern functional genomic techniques such as transgenesis in these worms. As it did for classical genetic study, the availability of a generation of free-living males and females of short duration (2-3 days) in the life cycles of Strongyloides spp. made it possible, nearly a decade ago, to translate standard methods for transgenesis in C. elegans to these parasites as well as the related genus Parastrongyloides $[54,9,10]$. Specifically, similarities in both the gross and fine structures of the gonads of $C$. elegans hermaphrodites and free-living female Strongyloides made it relatively easy to adapt methods for introducing transgene DNA constructs by gonadal microinjection of Strongyloides spp. (Fig. 1). Early on, studies in Strongyloides were limited to observations that could be made in transgenic worms of the first filial (F1) generation, owing to apparent silencing of transgenes encoded in standard plasmid vectors in the second filial (F2) generation and beyond [9]. Recently, however, we have learned that transgenes that are integrated into the chromosomes of $S$. ratti via the piggyBac transposon system are inherited stably and maintain their expression indefinitely through alternating rounds of host and culture passage [14•]. This advance resulted in establishment of the first stable transgenic lines of an animal parasitic nematode. In addition to piggy Bac-mediated chromosomal integration of transgenes, success in this effort hinged on the use of $S$. ratti, which, as a natural parasite of the rat, has a well-adapted rodent host in form of the laboratory rat. As all of our prior work on trangenesis had been done with $S$. stercoralis, we showed, preparatory to the study on piggyBac-mediated integration, that $S$. ratti could also be transformed by gonadal microinjection of plasmid-based vectors and that 5 and 3 regulatory sequences from $S$. stercoralis gave identical patterns of transgene expression in both $S$. stercoralis and $S$. ratti [11]. Potential applications of this approach to stable transgenesis in $S$. ratti and prospects for its translation to $S$. stercoralis are discussed in the Future Directions section.

\section{Nucleic Acid-Based Diagnosis}

Free Parasite Nucleic Acids in Diagnostic Specimens

Relative to parasitological diagnosis of other soil-transmitted human helminthiases, the sensitivity of single direct stool examination for larvae of $S$. stercoralis is notably low, and is only moderately improved by methods such as the Baermann technique and Koga's agar plate method, which involve amplification of parasite numbers by rearing through the freeliving generation [3]. In addition to the search for more reliable immunodiagnostic methods, the realization that parasite-specific free DNA is voided in the feces of human strongyloidiasis patients [55-60] has sparked keen interest in developing nucleic acid-based diagnostics for this parasitism.

Diagnostic Polymerase Chain Reaction Targets and Comparisons to Parasitological and Immune Diagnosis

PCR primer sets for detection of S. stercoralis-specific DNA in fecal samples are designed most often to amplify regions of the 18 s ribosomal RNA gene [56-58, 60]. Other genes targeted for this purpose in various assay schemes have been the internal transcribed spacer 1 (ITS1) region of the ribosomal RNA gene [55], a region of the 28s ribosomal RNA gene conserved in S. stercoralis and five additional Strongyloides species [59], the cytochrome c oxidase, subunit 1 gene [60], and the Strongyloides-specific repeat sequence AYO28262 [60]. In some cases these primer sets have been incorporated into multiplex PCR-based assays designed to detect not only $S$. stercoralis DNA, but that of other species of soiltransmitted helminth as well $[58,56]$. Where evaluations were done, PCR-based assays were virtually $100 \%$ specific for S. stercoralis $[58,59,57,60]$. When compared to direct fecal examination, PCR-based methods resulted in a two- to sevenfold increase in detection rates where any samples were positive by direct fecal examination [58, 60]. Similarly, a PCRbased method discovered three infected individuals among 400 patients that were negative by direct fecal examination [56]. However, when compared to more sensitive parasitological techniques for diagnosis of $S$. stercoralis infection, those involving culture amplification, real-time PCR targeting the 18 s ribosomal RNA gene proved highly sensitive with cultures containing high to medium numbers of parasites, but it detected only $15 \%$ of cultures with low (1-5 larvae) numbers of parasites per culture [57]. This underscores the reality that PCR is subject some of the same factors, such as discontinuous distribution of parasites (and perhaps parasite DNA) through the sample, that limit the sensitivity of direct microscopic fecal examination. Other factors that limit the sensitivity of PCR-based diagnosis of S. stercoralis and other enteric pathogens are the presence of inhibitory chemicals or viral DNA in stool [57]. An encouraging finding from the real-time 
PCR results in samples with low numbers of larvae per culture was that positive real-time PCR amplifications were characterized by higher $\mathrm{Ct}$ (cycle threshold) values than the results from cultures with moderate to high numbers of larvae per culture, thus indicating that real-time PCR has a semiquantitative capability that may be valuable in follow-up evaluations of mass chemotherapy programs.

\section{Future Directions}

Recent calls for heightened awareness of the global health impact of human strongyloidiasis and for its explicit incorporation into integrated control programs for soil-transmitted helminths $[2,1$, 3] should stimulate renewed interest in the epidemiology of S. stercoralis infection and of the factors predisposing chronic strongyloidiais patients to disseminated hyperinfection. A comprehensive genome project, comprising not only S. stercoralis, but also S. ratti, S. papillosus, S. venezuelensis and Parastrongyloides trichosuri, is nearing completion, and the annotated genomes of these parasites, with a comparative analysis of them, will be published in the very near future (Berriman et al., unpublished). It is also likely, and altogether warranted, that the increasing ease and decreasing cost of next-generation sequencing will allow RNAseq to come to the fore as the method of choice for transcriptomic comparisons of life stages of interest. It will be important for individual researchers to make data from such studies publicly available. One crucial transcriptomic comparison, which has not yet been undertaken to the author's knowledge, is between environmental L3i of S. stercoralis and L3i arising within susceptible hosts during the process of autoinfection. If a subset of genes regulated preferentially in L3a could be identified, these might contain potential targets for intervention to prevent the most serious complications of human strongyloidiasis. From a standpoint of basic developmental biology, products of such autoinfection-associated genes might represent essential controls over a process that is unique to $S$. stercoralis infection among all the soil-transmitted helmenthiases.

Recent advances in transgenesis in S. stercoralis and $S$. ratti, especially the new capability to prepare stable transgenic lines of parasite, should facilitate functional study of many of the putative 'genes for parasitism' identified in the transcriptomic studies discussed in this article. Gain-offunction studies, which would involve assessing phenotypes associated with over-expression of genes of interest, are possible with current techniques for transgenesis. Still lacking for Strongyloides spp. are loss-of-function methods such as RNA interference (RNAi) or specific gene disruption that have been proven to be such powerful functional genomic tools in other model organisms. One current method for gene editing that is now being applied in C. elegans is the CRISPR/Cas9 system, which allows precise targeting of gene sequences for insertion or deletion of key elements $[61 \bullet, 62,63]$. Methods for heritable transgenesis in $S$. ratti as discussed above open the formal possibility that this powerful technique can be applied to this species, and proof-of-principle for the CRISPR/Cas9 should be a top near-term research priority for the Strongyloides community. Similarly, augmentation of double-stranded RNA transport proteins in Strongyloides spp. by heterologous gene transfer from C. elegans is also formally possible and could constitute a means of boosting RNAi sensitivity, which is relatively low in members of this genus.

In the area of classical genetics, the new linkage map for $S$. ratti, which is discussed above, affords the ability to use genetic crosses to map phenotypic traits to specific loci in the genome. It will be of great interest to see this powerful forward genetic approach applied in the near future. Discovery of hostassociated chemoattractant molecules represents a significant advance in understanding the process of host finding and contact, a crucial prerequisite to the infective process in $S$. stercoralis. Application of new functional genomic methods to understand how these chemical cues are transduced in the worm will be a key priority in the near future. The trend towards application of nucleic acid-based diagnostic methods to human strongyloidiasis will likely increase in the near future. The recent discovery of parasite-specific microRNAs circulating in the plasma of hosts infected with schistosomes [64] and filariae [65] calls for investigation of the possibility that $S$. stercoralis-specific microRNAs also circulate in the plasma of human patients and could be diagnostic markers. Point-of-care diagnosis of $S$. stercoralis infection in human patients could be facilitated in the near future by application of loop-mediated isothermal amplification (LAMP) of parasite-specific nucleic acid markers. This method has already been investigated in other parasitic nematode infections $[66,67]$. Finally, the existence of parasitic and nonparasitic morphs (e.g.. parasitic and free-living females) with radically different body plans, ecological niches, lifespans, or developmental fates, all resulting from the same genotype, argues strongly for investigating epigenetic control of developmental fate in Strongyloides spp. Gene regulation at this level has not been studied extensively in parasitic nematodes, but recent work on platyhelminths, including schistosomes, indicates a clear role for epigenetic regulation of reproductive biology in those parasites [68-70].

Acknowledgments The author received support from grants R01 AI050668-09 (PI: JBL) and R21 AI105865 (Joint PI: JBL) from the US National Institutes of Health. The author is grateful to Dr. Jonathan Stoltzfus for helpful discussion.

\section{Compliance with Ethics Guidelines}

Conflict of Interest James B. Lok declares that he has no conflict of interest. 
Human and Animal Rights and Informed Consent This review article does not contain any unpublished studies with human or animal subjects performed by the author.

\section{References}

Papers of particular interest, published recently, have been highlighted as:

- Of importance

1. Greaves D, Coggle S, Pollard C, Aliyu SH, Moore EM. Strongyloides stercoralis infection. BMJ. 2013;347:f4610. doi:10. 1136/bmj.f4610.

2. Bisoffi Z, Buonfrate D, Montresor A, Requena-Mendez A, Munoz J, Krolewiecki AJ, et al. Strongyloides stercoralis: a plea for action. PLoS Negl Trop Dis. 2013;7(5):e2214. doi:10.1371/journal.pntd. 0002214.

3. Krolewiecki AJ, Lammie P, Jacobson J, Gabrielli AF, Levecke B, Socias E, et al. A public health response against Strongyloides stercoralis: time to look at soil-transmitted helminthiasis in full. PLoS Negl Trop Dis. 2013;7(5):e2165. doi:10.1371/journal.pntd. 0002165.

4. Mansfield LS, Niamatali S, Bhopale V, Volk S, Smith G, Lok JB, et al. Strongyloides stercoralis: maintenance of exceedingly chronic infections. Am J Trop Med Hyg. 1996;55(6):617-24.

5. Schad GA, Thompson F, Talham G, Holt D, Nolan TJ, Ashton FT, et al. Barren female Strongyloides stercoralis from occult chronic infections are rejuvenated by transfer to parasite-naive recipient hosts and give rise to an autoinfective burst. J Parasitol. 1997;83(5):785-91.

6. Caumes E, Keystone JS. Acute strongyloidiasis: a rarity. Chronic strongyloidiasis: a time bomb! J Travel Med. 2011;18(2):71-2.

7. Viney ME, Matthews BE, Walliker D. Mating in the nematode parasite Strongyloides ratti: proof of genetic exchange. Proc R Soc Lond B Biol Sci. 1993;254(1341):213-9.

8. Viney ME. A genetic analysis of reproduction in Strongyloides ratti. Parasitology. 1994;109(Pt 4):511-5.

9. Junio AB, Li X, Massey Jr HC, Nolan TJ, Todd Lamitina S, Sundaram MV, et al. Strongyloides stercoralis: cell- and tissuespecific transgene expression and co-transformation with vector constructs incorporating a common multifunctional 3' UTR. Exp Parasitol. 2008;118(2):253-65. doi:10.1016/j.exppara.2007.08. 018.

10. Li X, Massey HC, Nolan TJ, Schad GA, Kraus K, Sundaram M, et al. Successful transgenesis of the parasitic nematode Strongyloides stercoralis requires endogenous non-coding control elements. Int J Parasitol. 2006;36:671-9. doi:10.1016/j.ijpara.2005. 12.007 .

11. Li X, Shao H, Junio A, Nolan TJ, Massey Jr HC, Pearce EJ, et al. Transgenesis in the parasitic nematode Strongyloides ratti. Mol Biochem Parasitol. 2011;179(2):114-9. doi:10.1016/j.molbiopara. 2011.06.002.

12. Nemetschke L, Eberhardt AG, Hertzberg H, Streit A. Genetics, chromatin diminution, and sex chromosome evolution in the parasitic nematode genus Strongyloides. Curr Biol. 2010;20(19):168796. doi:10.1016/j.cub.2010.08.014.

13. Nemetschke L, Eberhardt AG, Viney ME, Streit A. A genetic map of the animal-parasitic nematode Strongyloides ratti. Mol Biochem Parasitol. 2010;169(2):124-7. The genetic map resulting from this work will support evaluation of sexual crosses designed to localize genetic traits of interest in $S$. ratti to delimited genomic regions.
This is a key component of a classical forward genetic approach, which is enabled by the presence of free-living males and females in the life cycles of Strongyloides spp.

14. Shao H, Li X, Nolan TJ, Massey Jr HC, Pearce EJ, Lok JB. Transposon-mediated chromosomal integration of transgenes in the parasitic nematode Strongyloides ratti and establishment of stable transgenic lines. PLoS Pathog. 2012;8(8):e1002871. doi:10. 1371/journal.ppat.1002871. This paper reports the first chromosomal integration of transgenes in Strongyloides spp., using the piggyBac transposon system. Most significantly, it reports the first establishment of lines of $S$. ratti that stably inherit and express a transgene construct. This advance is a prerequisite to applying methods such as the CRISPR/Cas9 system for genome editing.

15. Viney ME. Exploiting the life cycle of Strongyloides ratti. Parasitol Today. 1999;15(6):231-5. doi:10.1016/S0169-4758(99)01452-0.

16. Viney ME, Green LD, Brooks JA, Grant WN. Chemical mutagenesis of the parasitic nematode Strongyloides ratti to isolate ivermectin resistant mutants. Int J Parasitol. 2002;32(14):1677-82. doi:10. 1016/S0020-7519(02)00157-1.

17. Crook M. The dauer hypothesis and the evolution of parasitism: 20 years on and still going strong. Int J Parasitol. 2014;44(1):1-8. doi:10.1016/j.ijpara.2013.08.004.

18. Hotez P, Hawdon JM, Schad GA. Hookworm larval infectivity, arrest and amphiparatenesis: the Caenorhabditis elegans daf-c paradigm. Parasitol Today. 1993;9:23-6.

19. Stoltzfus JD, Bart SM, Lok JB. cGMP and NHR signaling co-regulate expression of insulin-like peptides and developmental activation of infective larvae in Strongyloides stercoralis. PLoS Pathog. 2014;10(7):e1004235. doi:10.1371/journal.ppat.1004235. This paper contains direct experimental evidence of the essential involvement of three cellular signaling pathways in developmental activation of cultured L3i of S. stercoralis. These pathways, GPCR signaling, ILS and steroid (dafachronic acid) hormone signaling, are crucial regulators of dauer larval development in $C$. elegans. The paper contains new and surprising findings indicating divergent epistatic relationships of the three types of signaling $S$. stercoralis.

20. Stoltzfus JD, Minot S, Berriman M, Nolan TJ, Lok JB. RNAseq analysis of the parasitic nematode Strongyloides stercoralis reveals divergent regulation of canonical dauer pathways. PLoS Negl Trop Dis. 2012;6(10):e1854. doi:10.1371/journal.pntd.0001854. This transcriptomic study created the most extensive RNAseq database to date for $S$. stercoralis. It contains sequences of the complete polyadenylated RNA repertoires of seven life stages of the parasite. This study also assessed trends in transcript abundance for genes homologous to those regulating dauer development in Caenorhabditis elegans. These support conservation of function in insulin-like signaling, GPCR signaling and steroid hormone signaling, but not TGF $\beta$-like signaling.

21. Zwaal RR, Mendel JE, Sternberg PW, Plasterk RH. Two neuronal G proteins are involved in chemosensation of the Caenorhabditis elegans dauer-inducing pheromone. Genetics. 1997;145(3):715-27.

22. Castelletto ML, Massey Jr HC, Lok JB. Morphogenesis of Strongyloides stercoralis infective larvae requires the DAF-16 ortholog FKTF-1. PLoS Pathog. 2009;5(4):e1000370. doi:10. 1371/journal.ppat.1000370.

23. Stoltzfus JD, Massey Jr HC, Nolan TJ, Griffith SD, Lok JB. Strongyloides stercoralis age-1: a potential regulator of infective larval development in a parasitic nematode. PLoS One. 2012;7(6): e38587. doi:10.1371/journal.pone.0038587.

24. Massey Jr HC, Ranjit N, Stoltzfus JD, Lok JB. Strongyloides stercoralis daf-2 encodes a divergent ortholog of Caenorhabditis elegans DAF-2. Int J Parasitol. 2013;43(7):515-20. doi:10.1016/j. ijpara.2013.01.008.

25. Motola DL, Cummins CL, Rottiers V, Sharma KK, Li T, Li Y, et al. Identification of ligands for DAF-12 that govern dauer formation 
and reproduction in C. elegans. Cell. 2006;124(6):1209-23. doi:10. 1016/j.cell.2006.01.037.

26. Wang Z, Zhou XE, Motola DL, Gao X, Suino-Powell K, Conneely A, et al. Identification of the nuclear receptor DAF-12 as a therapeutic target in parasitic nematodes. Proc Natl Acad Sci U S A. 2009;106(23):9138-43. doi:10.1073/pnas.0904064106.

27. Riddle DL, Albert PS. Genetic and Environmental Regulation of Dauer Larva Development. In: Riddle DL, Blumenthal T, Meyer BJ, Priess JR, editors. C. elegans II. 2nd ed. Cold Spring Harbor (NY)1997.

28. Silva CV, Goncalves AL, Cruz L, Cruz MC, Ueta MT, Costa-Cruz JM. F-actin accumulates in the vulva of female Strongyloides venezuelensis. J Helminthol. 2013;87(3):301-4. doi:10.1017/ S0022149X12000351.

29. Machado ER, Ueta MT, Lourenco EV, Anibal Fde F, Silva EV, Roque-Barreira MC, et al. Infectivity of Strongyloides venezuelensis is influenced by variations in temperature and time of culture. Exp Parasitol. 2011;127(1):72-9. doi:10.1016/j.exppara. 2010.06.027

30. Charlesworth B. Sex determination: a worm does it by elimination. Curr Biol. 2010;20(19):R841-3. doi:10.1016/j.cub.2010.08.032.

31. Safer D, Brenes M, Dunipace S, Schad G. Urocanic acid is a major chemoattractant for the skin-penetrating parasitic nematode Strongyloides stercoralis. Proc Natl Acad Sci U S A. 2007;104(5):1627-30.

32. Castelletto ML, Gang SS, Okubo RP, Tselikova AA, Nolan TJ, Platzer EG, et al. Diverse host-seeking behaviors of skinpenetrating nematodes. PLoS Pathog. 2014;10(8):e1004305. doi: 10.1371/journal.ppat.1004305.

33. Streit A. Reproduction in Strongyloides (Nematoda): a life between sex and parthenogenesis. Parasitology. 2008;135(3):285-94. doi: 10.1017/S003118200700399X.

34. O'Meara H, Barber R, Mello LV, Sangaralingam A, Viney ME, Paterson S. Response of the Strongyloides ratti transcriptome to host immunological environment. Int J Parasitol. 2010;40(14): 1609-17. doi:10.1016/j.ijpara.2010.06.005.

35. Blaxter ML, De Ley P, Garey JR, Liu LX, Scheldeman P, Vierstraete A, et al. A molecular evolutionary framework for the phylum Nematoda. Nature. 1998;392(6671):71-5. doi:10.1038/ 32160

36. Marcilla A, Garg G, Bernal D, Ranganathan S, Forment J, Ortiz J, et al. The transcriptome analysis of Strongyloides stercoralis L3i larvae reveals targets for intervention in a neglected disease. PLoS Negl Trop Dis. 2012;6(2):e1513. doi:10.1371/journal.pntd. 0001513

37. Nagayasu E, Ogura Y, Itoh T, Yoshida A, Chakraborty G, Hayashi $\mathrm{T}$, et al. Transcriptomic analysis of four developmental stages of Strongyloides venezuelensis. Parasitol Int. 2013;62(1):57-65. doi: 10.1016/j.parint.2012.09.006.

38. Yoshida A, Nagayasu E, Nishimaki A, Sawaguchi A, Yanagawa S, Maruyama H. Transcripts analysis of infective larvae of an intestinal nematode, Strongyloides venezuelensis. Parasitol Int. 2011;60(1):75-83. doi:10.1016/j.parint.2010.10.007.

39. Thompson FJ, Barker GL, Nolan T, Gems D, Viney ME. Transcript profiles of long- and short-lived adults implicate protein synthesis in evolved differences in ageing in the nematode Strongyloides ratti. Mech Ageing Dev. 2009;130(3):167-72. doi:10.1016/j.mad. 2008.11.001.

40. Ramanathan R, Varma S, Ribeiro JM, Myers TG, Nolan TJ, Abraham D, et al. Microarray-based analysis of differential gene expression between infective and noninfective larvae of Strongyloides stercoralis. PLoS Negl Trop Dis. 2011;5(5):e1039. doi:10.1371/journal.pntd.0001039.

41. Spinner WG, Thompson FJ, Emery DC, Viney ME. Characterization of genes with a putative key role in the parasitic lifestyle of the nematode Strongyloides ratti. Parasitology.
2012;139(10):1317-28. doi:10.1017/S0031182012000637. This paper represents the culmination of a series of studies dating to 2005 that have aimed to identify genes whose patterns of expression suggest that they are important in initiation and maintenance of the parasitic state in S. ratti. It represents the first systematic and unbiased screening approach to identifying 'genes for parasitism' in Strongyloides and identifies three leads in the search for new vaccine and drug targets.

42. Thompson FJ, Barker GL, Hughes L, Viney ME. Genes important in the parasitic life of the nematode Strongyloides ratti. Mol Biochem Parasitol. 2008;158(2):112-9. doi:10.1016/j.molbiopara. 2007.11.016.

43. Thompson FJ, Mitreva M, Barker GL, Martin J, Waterston RH, McCarter JP, et al. An expressed sequence tag analysis of the lifecycle of the parasitic nematode Strongyloides ratti. Mol Biochem Parasitol. 2005;142(1):32-46. doi:10.1016/j.molbiopara.2005.03. 006.

44. Wilkes CP, Bleay C, Paterson S, Viney ME. The immune response during a Strongyloides ratti infection of rats. Parasite Immunol. 2007;29(7):339-46.

45. Biewener V, Welz C, Khumpool G, Kuttler U, Schnieder T. Strongyloides papillosus: changes in transcript levels of lysozyme and aspartic protease 2 in percutaneously migrated larvae. Exp Parasitol. 2012;132(1):62-8. doi:10.1016/j.exppara.2012.02.009.

46. Mello LV, O'Meara H, Rigden DJ, Paterson S. Identification of novel aspartic proteases from Strongyloides ratti and characterisation of their evolutionary relationships, stage-specific expression and molecular structure. BMC Genomics. 2009;10:611. doi:10. 1186/1471-2164-10-611.

47. Mulvenna J, Hamilton B, Nagaraj SH, Smyth D, Loukas A, Gorman JJ. Proteomics analysis of the excretory/secretory component of the blood-feeding stage of the hookworm. Ancylostoma Caninum Mol Cell Proteomics. 2009;8(1):109-21. doi:10.1074/ mcp.M800206-MCP200.

48. Baugh LR, Kurhanewicz N, Sternberg PW. Sensitive and precise quantification of insulin-like mRNA expression in Caenorhabditis elegans. PLoS One. 2011;6(3):e18086. doi:10.1371/journal.pone. 0018086.

49. Nagayasu E, Ishikawa SA, Taketani S, Chakraborty G, Yoshida A, Inagaki $\mathrm{Y}$, et al. Identification of a bacteria-like ferrochelatase in Strongyloides venezuelensis, an animal parasitic nematode. PLoS One. 2013;8(3):e58458. doi:10.1371/journal.pone.0058458.

50. Soblik H, Younis AE, Mitreva M, Renard BY, Kirchner M, Geisinger F, et al. Life cycle stage-resolved proteomic analysis of the excretome/secretome from Strongyloides ratti-identification of stage-specific proteases. Mol Cell Proteomics. 2011;10(12):M111 010157. doi:10.1074/mcp.M111.010157. This study incorporates the first modern proteomic approach to characterize the secreted proteins of iL3, parasitic females and free-living stages of $S$. ratti. Its findings include a remarkable concentration of secreted protein expression in $i L 3$, the stage of the parasite that initiates host infection by penetrating skin. It also demonstrates a potentially essential function of a prolyl oligopeptidase in promoting survival by parasitic female worms.

51. Younis AE, Geisinger F, Ajonina-Ekoti I, Soblik H, Steen H, Mitreva M, et al. Stage-specific excretory-secretory small heat shock proteins from the parasitic nematode Strongyloides rattiputative links to host's intestinal mucosal defense system. Febs J. 2011;278(18):3319-36. doi:10.1111/j.1742-4658.2011.08248.x.

52. Garg G, Ranganathan S. In silico secretome analysis approach for next generation sequencing transcriptomic data. BMC Genomics. 2011;12 Suppl 3:S14. doi:10.1186/1471-2164-12-S3-S14.

53. Valentim CL, Cioli D, Chevalier FD, Cao X, Taylor AB, Holloway SP, et al. Genetic and molecular basis of drug resistance and species-specific drug action in schistosome parasites. Science. 2013;342(6164):1385-9. doi:10.1126/science.1243106. 
54. Grant WN, Skinner SJM, Howes JN, Grant K, Shuttleworth G, Heath DD, et al. Heritable transgenesis of Parastrongyloides trichosuri: a nematode parasite of mammals. Int J Parasitol. 2006;36:475-83. doi:10.1016/j.ijpara.2005.12.002.

55. Ahmad AF, Hadip F, Ngui R, Lim YA, Mahmud R. Serological and molecular detection of Strongyloides stercoralis infection among an Orang Asli community in Malaysia. Parasitol Res. 2013;112(8): 2811-6. doi:10.1007/s00436-013-3450-z.

56. Mejia R, Vicuna Y, Broncano N, Sandoval C, Vaca M, Chico M, et al. A novel, multi-parallel, real-time polymerase chain reaction approach for eight gastrointestinal parasites provides improved diagnostic capabilities to resource-limited at-risk populations. Am J Trop Med Hyg. 2013;88(6):1041-7. doi:10.4269/ajtmh.12-0726.

57. Sultana Y, Jeoffreys N, Watts MR, Gilbert GL, Lee R. Real-time polymerase chain reaction for detection of Strongyloides stercoralis in stool. Am J Trop Med Hyg. 2013;88(6):1048-51. doi:10.4269/ ajtmh.12-0437.

58. Basuni M, Muhi J, Othman N, Verweij JJ, Ahmad M, Miswan N, et al. A pentaplex real-time polymerase chain reaction assay for detection of four species of soil-transmitted helminths. Am J Trop Med Hyg. 2011;84(2):338-43. doi:10.4269/ajtmh.2011.10-0499.

59. Kramme S, Nissen N, Soblik H, Erttmann K, Tannich E, Fleischer $\mathrm{B}$, et al. Novel real-time PCR for the universal detection of Strongyloides species. J Med Microbiol. 2011;60(Pt 4):454-8. doi:10.1099/jmm.0.025338-0.

60. Verweij JJ, Canales M, Polman K, Ziem J, Brienen EA, Polderman $\mathrm{AM}$, et al. Molecular diagnosis of Strongyloides stercoralis in faecal samples using real-time PCR. Trans R Soc Trop Med Hyg. 2009;103(4):342-6. doi:10.1016/j.trstmh.2008.12.001.

61. Friedland AE, Tzur YB, Esvelt KM, Colaiacovo MP, Church GM, Calarco JA. Heritable genome editing in C. elegans via a CRISPRCas9 system. Nat Methods. 2013;10(8):741-3. doi:10.1038/nmeth. 2532. This paper reports adaptation of the CRISPR/Cas9 system for genome editing to the free-living nematode C. elegans. CRISPR/ Cas9 is the most powerful and efficient method to date for making insertions and deletions into specific gene loci. Plasmid constructs reported here for expression of the Cas 9 endonuclease and for single-guide RNAs in C. elegans are readily adaptable to the systems transgenesis in Strongyloides spp. that have been reported recently.

62. Tzur YB, Friedland AE, Nadarajan S, Church GM, Calarco JA, Colaiacovo MP. Heritable custom genomic modifications in Caenorhabditis elegans via a CRISPR-Cas9 system. Genetics. 2013;195(3):1181-5. doi:10.1534/genetics.113.156075.

63. Waaijers S, Portegijs V, Kerver J, Lemmens BB, Tijsterman M, van den Heuvel S, et al. CRISPR/Cas9-targeted mutagenesis in Caenorhabditis elegans. Genetics. 2013;195(3):1187-91. doi:10. 1534/genetics.113.156299.

64. Hoy AM, Lundie RJ, Ivens A, Quintana JF, Nausch N, Forster T, et al. Parasite-derived microRNAs in host serum as novel biomarkers of helminth infection. PLoS Negl Trop Dis. 2014;8(2): e2701. doi:10.1371/journal.pntd.0002701.

65. Tritten L, Burkman E, Moorhead A, Satti M, Geary J, Mackenzie C, et al. Detection of circulating parasite-derived microRNAs in filarial infections. PLoS Negl Trop Dis. 2014;8(7):e2971. doi:10.1371/ journal.pntd.0002971.

66. Li X, Liu W, Wang J, Zou D, Wang X, Yang Z, et al. Rapid detection of Trichinella spiralis larvae in muscles by loopmediated isothermal amplification. Int J Parasitol. 2012;42(1314):1119-26. doi:10.1016/j.ijpara.2012.09.011.

67. Takagi H, Itoh M, Kasai S, Yahathugoda TC, Weerasooriya MV, Kimura E. Development of loop-mediated isothermal amplification method for detecting Wuchereria bancrofti DNA in human blood and vector mosquitoes. Parasitol Int. 2011;60(4):493-7. doi:10. 1016/j.parint.2011.08.018.

68. Geyer KK, Chalmers IW, Mackintosh N, Hirst JE, Geoghegan R, Badets $\mathrm{M}$, et al. Cytosine methylation is a conserved epigenetic feature found throughout the phylum Platyhelminthes. BMC Genomics. 2013;14:462. doi:10.1186/1471-2164-14-462.

69. Geyer KK, Rodriguez Lopez CM, Chalmers IW, Munshi SE, Truscott M, Heald J, et al. Cytosine methylation regulates oviposition in the pathogenic blood fluke Schistosoma mansoni. Nat Commun. 2011;2:424. doi:10.1038/ncomms1433.

70. Lepesant JM, Grunau C, Cosseau C. Towards an understanding of the epigenetics of schistosomes: a comparative epigenomic study. Mem Inst Oswaldo Cruz. 2011;106(7):823-30. 